\title{
Overexpression of HIC1 plays a protective effect on renal cell injury caused by lipopolysaccharide by inhibiting IL-6/STAT3 pathway
}

\author{
Xiang Yang ${ }^{1}$, Jianfeng Gong ${ }^{1}$, Xiaosheng Cai ${ }^{2}$, Youjun Yuan ${ }^{2}$,
}

\begin{abstract}
${ }^{1}$ Department of Intensive care unit 2, Qingyuan People's Hospital, 511518 Qingyuan, Guangdong, China

${ }^{2}$ Department of Emergency, Wenzhou Central Hospital, 325000 Wenzhou, Zhejiang, China
\end{abstract}

\section{*Correspondence}

you_junyuan@163.com

(Youjun Yuan)

\begin{abstract}
Sepsis is a life-threatening condition that can even occur due to an infection. Systemic inflammatory response syndrome acts a pivotal role in acute kidney injury (AKI). Although great advancements have been achieved for treating sepsis-induced AKI, its prognosis and pathophysiology remain unclear. In order to gain insights into the relevant role of hypermethylated in cancer 1 ( $\mathrm{HICl}$ ) in AKI, a cellular model of AKI caused by sepsis was performed in lipopolysaccharide (LPS)-treated human kidney 2 (HK-2) cell line. The overexpression vector pcDNA3.1-HICl was transfected into HK-2 cell line to examine the effects of $\mathrm{HICl}$ on LPS-treated HK-2 cell line. Reverse transcriptionquantitative polymerase chain reaction (RT-qPCR), western blot and enzyme-linked immunosorbent assay (ELISA) assays were performed to examine the alterations in the expression levels of $\mathrm{HICl}$, cell apoptosis, or inflammation-related biomarkers. The apoptotic rate of HK-2 cell line was measured by flow cytometry. This study suggested that LPS treatment downregulated $\mathrm{HICl}$ and inhibited $\mathrm{HK}-2$ cell viability, whereas $\mathrm{HICl}$ overexpressing reversed these effects. Importantly, $\mathrm{HICl}$ has a protective effect on LPSinduced cellular apoptosis and inflammatory response. Moreover, overexpression of HIC1 suppressed the LPS-induced activation of IL-6/STAT3 signaling pathway in HK-2 cell line. HICl protects HK-2 cell line against LPS-induced damage, which was partly through the inhibition of IL-6/STAT3 signaling pathways.
\end{abstract}

\section{Keywords}

Sepsis; Renal cell injury; HIC1 cell line; IL-6/STAT3; LPS

\section{Introduction}

Sepsis is a disease caused by an infection, usually with a severe systemic inflammatory response, resulting in various organ dysfunction, and its mortality rate is high, especially in elderly $[1,2]$. Acute kidney injury (AKI) is a serious and lethal complication of the sepsis process, represented by insufficient blood filtration, imbalance of water and ions, and damaged urine production $[3,4]$. Numerous studies have shown that systemic inflammatory response syndrome act a pivotal role in AKI $[5,6]$. The inflammatory factors such interleukin 6 (IL-6), inducible nitric oxide synthase (iNOS), interleukin 1 beta (IL-1 $\beta$ ), and tumor necrosis factor $\alpha$ (TNF- $\alpha$ ) were reported as crucial immune response factors which occur and damage the kidney [7]. Recent researches have demonstrated that the cellular apoptosis, inflammatory response, and the expression levels of oxidative stress in renal tubular cells may be associated with the pathological process of AKI $[8,9]$. Although great advancements have been achieved for treating sepsis-induced AKI, its prognosis and pathophysiology remain unclear.
Hypermethylated in Cancer 1 (HICl) is a tumor suppressor gene located at $17 \mathrm{p} 13.3$ [10], which could regulate target genes, including fibroblast growth factor binding protein 1 , cyclin D1, cyclin dependent kinase inhibitor $1 \mathrm{C}$ and $\mathrm{p} 21$, which were involved in the occurrence and progression of various tumors [11-13]. A recent study has indicated that $\mathrm{HICl}$ reduced cell invasion and metastasis by suppressing the IL-6/STAT3 signaling pathway in human pancreatic cancer [14]. Another study has also reported that $\mathrm{HICl}$ regulated high glucose-induced reactive oxygen species (ROS) accumulation in renal tubular epithelial cells through epigenetic inhibition of silent information regulator 1 (SIRT1) transcription [15]. However, there are rare reports considering the role of $\mathrm{HICl}$ in AKI caused by sepsis.

The signal transducers and activators of transcription (STAT) family proteins can regulate principal cellular progression, including cell proliferation, differentiation, inflammatory response, angiogenesis, and metastasis [16]. The STAT signal transduction pathway can be activated in response to many protein ligands, including cytokines, growth factors, and interferons $[17,18]$. In particular, STAT3 acts as 
a transcription factor for oncogenes and mediates oncogenic transformation [19]. Excessive inflammatory response and cytokine storm cause serious damage to multiple organs. IL-6, TNF- $\alpha$, IL- $1 \beta$ are important inflammatory factors that are released when the immune response occurs and cause damage to the kidney. IL- 6 is a key cytokine for immune response and tumorigenesis [20], and IL-6 and its main factor STAT3 are also well-known as tumor-promoting factors in numerous cancers [21]. A previous study reported that adiponectin could attenuated the expression of inflammatory factors TNF- $\alpha$ and IL-6 through the IL-6/STAT3 signaling pathway, accordingly suppressing lung injury caused by sepsis [22] Furthermore, STAT3 is one of the proteins associated with HIC1, which can inhibit the activity of related molecules and regulate the expression of target gene mediated by STAT3 and involved in the cellular biological functions [23]. Thus, this study investigated the role of HICl in regulating IL-6/STAT3 signaling pathway, which thus participates in sepsis-induced renal cell injury.

\section{Methods}

\subsection{Cell culture}

Human renal proximal tubular epithelial cell line (HK-2) was purchased from American Type Culture Collection (Manassas, WV, USA) and maintained in DMEM/Ham's F12 growth medium (Gibco, Waltham, MA, USA) containing fetal bovine serum $(10 \%$, Gibco) and penicillin/streptomycin (1\%). HK-2 cell line was maintained $\left(37^{\circ} \mathrm{C}, 5 \%\right.$ Carbon dioxide, $\left.\mathrm{CO}_{2}\right)$ and pre-transfected with pcDNA3.1 or pcDNA3.1 HIC1 at 24-48 hours before treatment of LPS $(5 \mu \mathrm{g} / \mathrm{mL})$.

\section{2 qPCR analysis}

RNA was obtained from HK-2 cell line using TRIzol method (Invitrogen, Carlsbad, CA, USA). The Prime-Script RT reagent kit (Tiangen, Beijing, China) was used to synthesize cDNA. QRT-PCR was performed on an ABI 7500 real-time PCR system (Applied Biosystems, Foster City, CA, USA) and detected by using SYBR Green PCR Master Mix (TaKaRa, Dalian, China).

The primers were as follow: HIC1: Forward (F): 5'-GAGTTGGGGAAGAGATGTGGAG-3'; reverse ( ${ }^{\prime}$ 5'-CCAACCCCAAATACTCCTAAACA'3', TNF- $\alpha$ : F' 5'-AGGACACCATGAGCACTGA'3'; R' 5'-CCGATCACTCCAAAGTGCA'-3', IL1 $\beta$ : F' 5'-CTCTCTCCTTTCAGGGCCA'-3'; $\quad$ R' $^{\prime}$ 5'-GCGGTTGCTCATCAGAATG'-3', IL-6: F' 5'-ATGAACTCCTTCTCCACAAG'-3'; R' 5'CTACATTTGCCGAAGAGCCCTCAGGCTGGACT'-3', GAPDH: F' 5'-TCTTTTGCGT CGCCAGCCGA'-3'; R' 5'-TGACCAGGCG CCCAATACGA'-3'.

\subsection{Western blot}

HK-2 cell line was collected using RIPA buffer and centrifuged (13,000 g, $4{ }^{\circ} \mathrm{C}, 15$ minutes). Protein samples $(20 \mu \mathrm{g})$ were separated by sodium dodecyl sulfate-polyacrylamide gel electrophoresis (SDS-PAGE) gel $(8 \%)$ and then transferred to nitrocellulose membranes (Bio-Rad, Hercules, CA, USA). The primary antibodies against HIC1 (1:2000, Santa Cruz Biotechnology, Inc. (SCB), Dallas, TX, USA), $\beta$-actin (1:10,000, SCB), BCL2 Associated X (Bax) (1:2000, Abcam, Cambridge, MA, USA), Cleaved Caspase 3 (1:2000, SCB), STAT3 (1:2000, Cell Signaling Technology (CST), Beverly, MA, USA), phosphorylation of Signal Transducer And Activator Of Transcription 3 (p-STAT3) (1:1000, CST), IL-6 (1:2000, Abcam), RAR-related orphan receptor gamma $(\mathrm{ROR} \gamma \mathrm{t})(1: 2000, \mathrm{SCB})$, Suppressor Of Cytokine Signaling 3 (SOCS3) (1:2000, CST) and B-cell lymphoma 2 (Bcl-2) (1:2000, Abcam) overnight $\left(4{ }^{\circ} \mathrm{C}\right)$. Horseradish peroxidase-labeled secondary antibody was used to incubate the membrane $\left(37^{\circ} \mathrm{C}, 1\right.$ hour). The proteins on the membrane were visualized by the enhanced chemiluminescence (SigmaAldrich, St. Louis, MO, USA). $\beta$-actin was used as the internal control.

\subsection{MTT assay}

After pre-transfected with pcDNA3.1 or pcDNA3.1 HICl at 24-48 hours before treatment of LPS $(1 \mu \mathrm{g} / \mathrm{mL})$, HK-2 cell line was seeded $\left(5 \times 10^{4} /\right.$ well, 96 -well plate, 24 hours $)$, and incubated $(10 \mu \mathrm{L}, 5 \mathrm{mg} / \mathrm{mL}$, MTT; Sigma, St. Louis, MO, USA). After incubation ( 4 hours, $37^{\circ} \mathrm{C}$ ), solubilized the formazan crystals in DMSO. The microplate reader was used to detect the absorbance $(570 \mathrm{~nm})$.

\subsection{Enzyme-linked immunosorbent assay (ELISA)}

The expression levels of TNF- $\alpha$, IL- 6 , and IL- $1 \beta$ in the supernatant from treated HK-2 cell line were measured using ELISA (R\&D Systems, Minneapolis, MN, USA).

\subsection{Flow cytometry}

The flow cytometry Annexin V-FITC/PI apoptosis detection kit was used to determine the apoptosis in HK-2 cell line. Cells were harvested and resuspended at a concentration of $10^{6}$ cells/mL. FITC-Annexin V and PI were added and kept on ice (in the dark, 15 minutes). The ratio of apoptotic cells was detected by flow cytometry.

\subsection{Statistics}

All experiments were obtained in triplicate and analyzed using GraphPad Prism Software 6.0 (GraphPad Software, La Jolla, CA, USA). All data were presented as mean \pm standard error of the mean (SEM). One-way analysis of variance (ANOVA) followed by Bonferroni's post-hoc test was used for comparison. A statistically significant difference was defined as a $p<$ 0.05 .

\section{Results}

\subsection{Overexpression of HIC1 promotes the viability of LPS treated HK-2 cell line}

LPS, a widely conceded stimulator of cellular apoptosis and inflammatory response, was used to treated HK-2 cell 
A

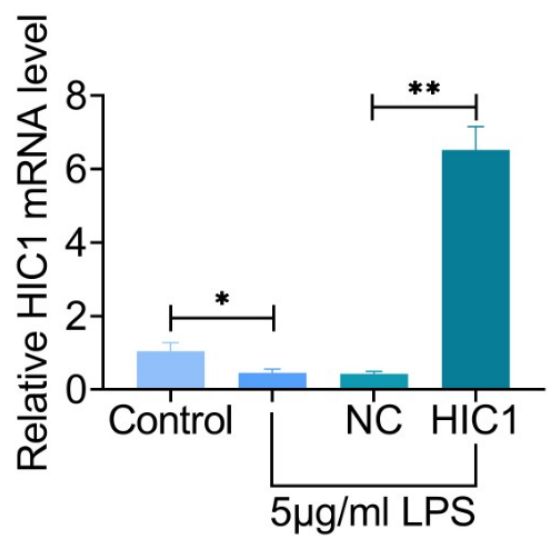

C

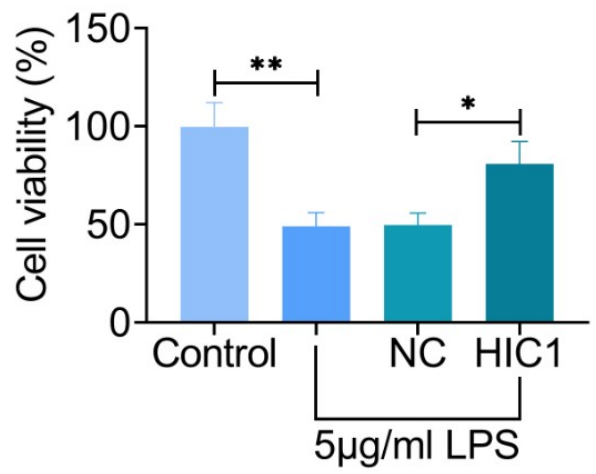

HIC1

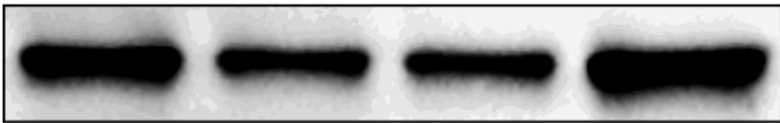

$\beta$-actin

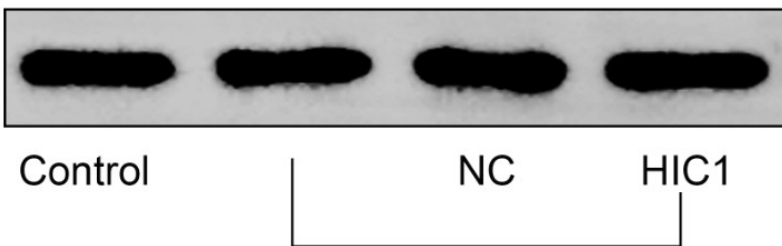

$5 \mu \mathrm{g} / \mathrm{ml}$ LPS

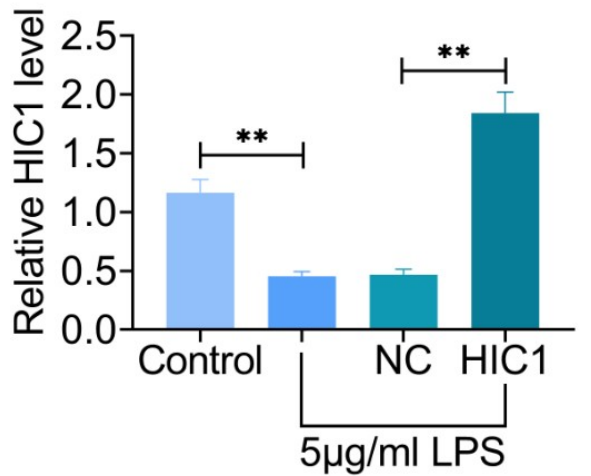

F I G U R E 1. Effects of HIC1 on the LPS-induced viability in HK-2 cell line, HK-2 cell line was transfected with control vector (pcDNA3.1) (NC) or HIC1 overexpression vector, followed by LPS treatment. (A) HICl expression was determined by qPCR. (B) Western blot analysis and quantitative data of $\mathrm{HICl}$ expression in LPS-treated HK-2 cell line. (C) Cell viability was estimated using MTT assay $* p<0.05$ and ${ }^{* *} p<0.01 v s$. control or LPS + HIC, data are expressed as mean \pm SEM.

line to establish a cellular model of AKI caused by sepsis. QPCR and western blot assays showed that HIC1 expression was remarkably suppressed in HK-2 cell line upon LPS treatment (Fig. 1A,B). HK-2 cell line that transfected with HICl overexpressing vector remarkably increased the HICl expression levels compared with pcDNA3.1 transfection (NC group) (Fig. 1A,B). In addition, the viability of LPS treated HK-2 cell line was significantly raised after transfection of HICl overexpressing vector (Fig. 1C). These data indicated that LPS downregulated $H I C l$ expression and reduced the viability in HK-2 cell line, and HICl overexpressing vector could reverse the effects induced by LPS.

\subsection{Overexpression of HIC1 suppressed LPS-induced apoptosis in HK-2 cell line}

The flow cytometry was performed to identify the regulatory effect of HICl overexpression on cellular apoptosis. The number of apoptosis-positive cells was remarkably increased in the LPS group as compared to the control group, while decreased by HICl overexpression. Similar findings were detected by western blot, revealed by determining the expression levels of apoptosis-related biomarkers in HK-2 cell line
(Fig. 2A). As presented in Fig. 2B, the expression levels of Bax and Caspase 3 in LPS treated HK-2 cell line were markedly increased compared with the control group, while decreased after transfected with HIC1 overexpressing vector. Moreover, LPS treatment induced a reduction in the Bcl-2 expression, which was enhanced by transfection with $\mathrm{HICl}$ overexpressing vector. These results implied that $H I C 1$ might be involved in the pathophysiological processes in LPS-induced cellular apoptosis.

\subsection{LPS-induced inflammation in HK-2 cell line was suppressed by overexpression of HIC1}

QPCR assay indicated that the mRNA expression levels of proinflammatory cytokines (IL-6, TNF- $\alpha$, and IL- $1 \beta$ ) in HK-2 cell line were significantly increased by LPS treatment (Fig. 3A), while transfection of $\mathrm{HICl}$ overexpression vector attenuated LPS-induced proinflammatory cytokine gene expressions in HK-2 cell line. In addition, ELISA assay verified that the protein expression levels of proinflammatory cytokines were increased by LPS treatment. Transfection of HIC1 overexpression vector in LPS-treated HK-2 cell line 
A
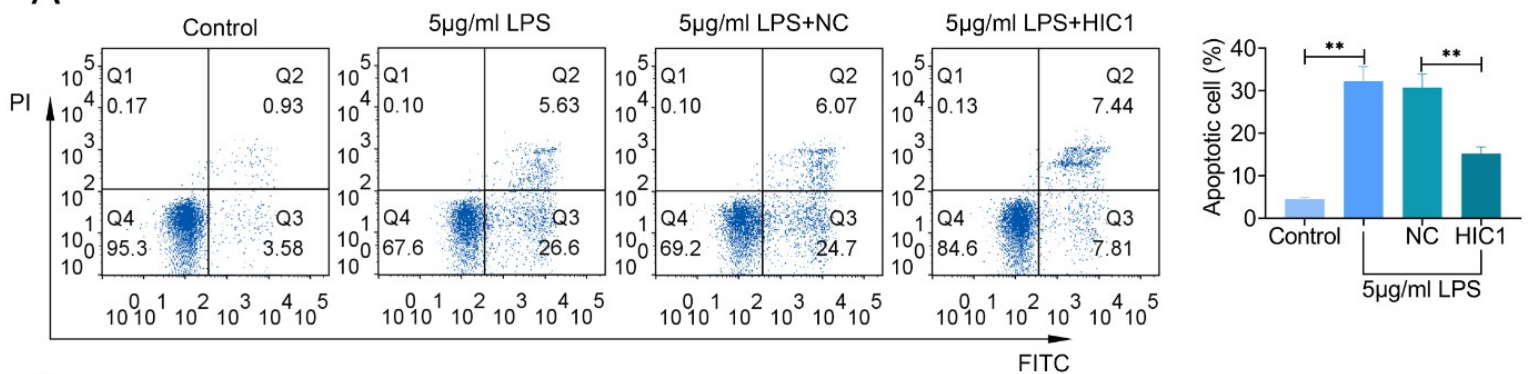

B
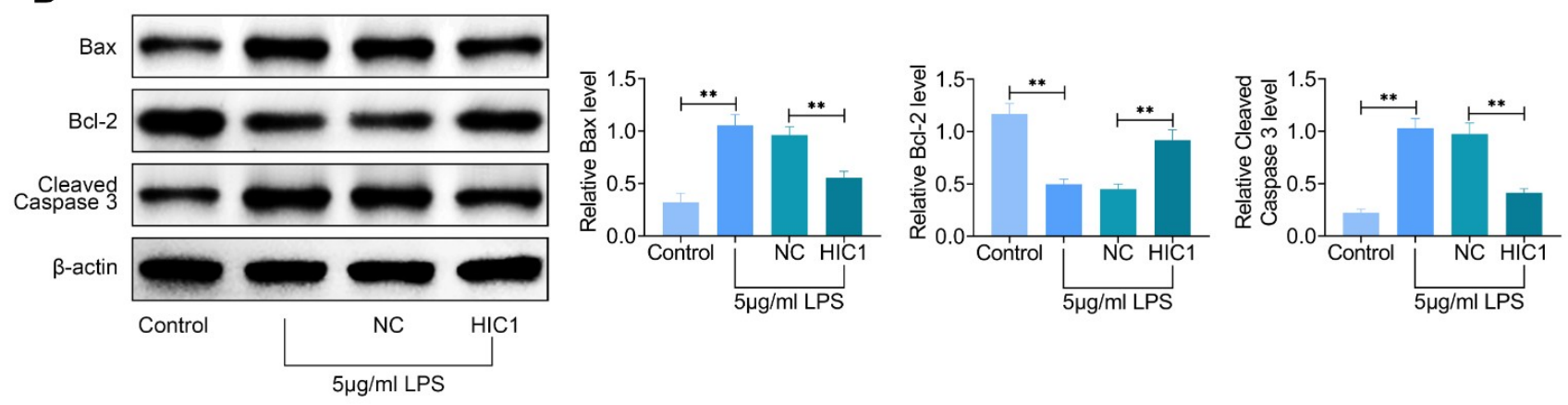

F IGURE 2. Overexpression of HIC1 suppressed apoptosis in HK-2 cell line induced by LPS. (A) The apoptotic rate of HK-2 cell line was measured by flow cytometry. (B) The protein expressions of Bax, Cleaved Caspase 3, and Bcl-2 were examined by Western blot analysis. ${ }^{*} p<0.05$ and ${ }^{* *} p<0.01 v s$. control or LPS + HIC. Data are expressed as mean \pm SEM.

obviously reversed these results (Fig. 3B). Together, these data supported a role of $\mathrm{HICl}$ in mediating the regulatory role of LPS in inflammatory response.

\subsection{Overexpression of HIC1 suppressed LPS-induced activation of IL-6/STAT3 signaling pathway in HK-2 cell line}

IL-6/STAT3 signaling pathway has been reported closely related to the enhanced inflammatory response, further study was explored its involvement in the regulation of $\mathrm{HICl}$ in LPStreated HK-2 cell line. Western blot analysis revealed that LPS activated the protein expressions of STAT3, p-STAT3, IL6, and ROR $\gamma$ t. Conversely, the expression level of SOCS3, related to the function of anti-inflammatory, was significantly decreased in LPS-treated HK-2 cell line. Transfection of $\mathrm{HICl}$ overexpression vector could reverse the simulative effect of LPS on IL-6/STAT3 signaling pathway (Fig. 4). These results highlighted that $\mathrm{HICl}$ exerted a protective influence on LPS-induced renal cell injury by suppressing the IL-6/STAT3 signaling pathway.

\section{Discussion}

AKI is one of common complications of sepsis and associated with renal inflammation, whose main characteristics are acute renal dysfunction. Multiple evidences have indicated that AKI is precipitated by unique and complex mechanisms, including abnormal kidney damage biomarkers, such as insulinlike growth factor-binding protein 7, interleukin-18 and L-type fatty acid binding protein [24]. LPS is a classic TLR4 agonist commonly used to induce sepsis in an animal model, which can cause a direct and consequential inflammatory response, thus stimulating the activation of the innate immune system in sepsis. The molecular mechanism of $\mathrm{HICl}$ as a tumor suppressor is to suppress tumor development by regulating the interactions between its target genes or proteins [25]. Previous studies have reported that $\mathrm{HICl}$ was constantly epigenetically silenced or deleted in prevalent human cancers [10,26]. Despite extensive investigations and the determination that $\mathrm{HICl}$ expression is detectable in a wide range of cells, there are no studies on the molecular basis of HICl inhibition of sepsis-induced AKI progression. Here, the present study initially found that $\mathrm{HICl}$ expression was notably downregulated after LPS treatment and the overexpression of $\mathrm{HICl}$ significantly improved the viability in LPS-treated HK-2 cell line. Previous reports have shown that overexpression of $\mathrm{HICl}$ was related to tumor proliferation, migration, apoptosis and invasion $[25,27]$. In the present study, its anti-apoptotic effect was demonstrated through two different kinds of experiments. The results from flow cytometry assay indicated that the number of apoptosis-positive cell was significantly increased in the LPS treatment group, while overexpression of $\mathrm{HICl}$ reversed these results. Moreover, the results from western blot demonstrated similar results. Though a recent research has reported that hypermethylation of $\mathrm{HICl}$ promoter and overexpression of $\mathrm{HICl}$ protein appear in some cancer cells [28], this is the first report showing that $\mathrm{HICl}$ overexpression was correlated with LPS-induced acute renal cell dysfunction. LPS induces mitochondrial dysfunction and inflammation in multiple cell types and is frequently used to develop renal or intestine injury models. LPS mediates cellular apoptogenic signals via the release of cytochrome $\mathrm{C}$ and alterations in the mitochondrial membrane potential [29]. 
A

B
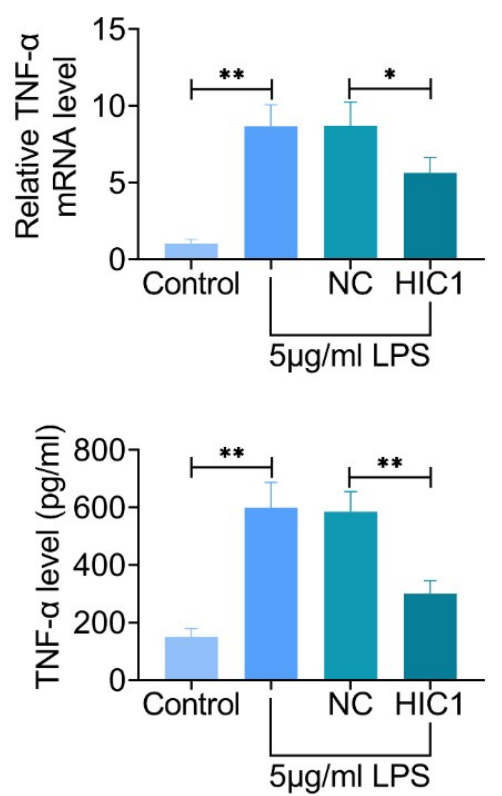
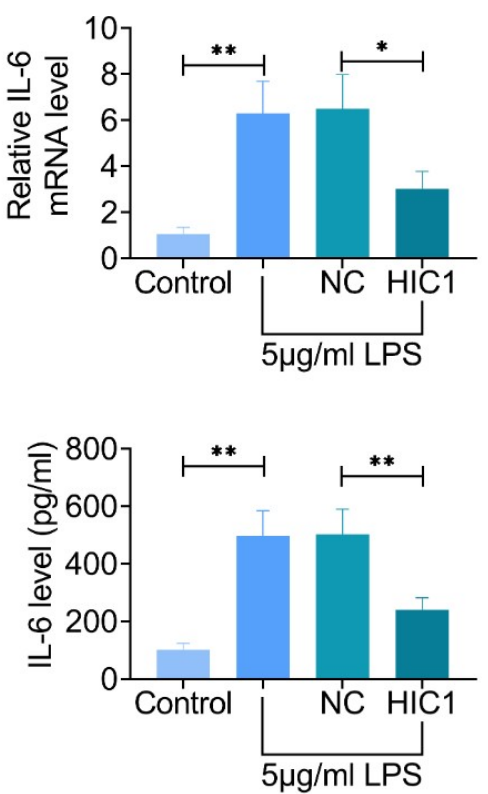
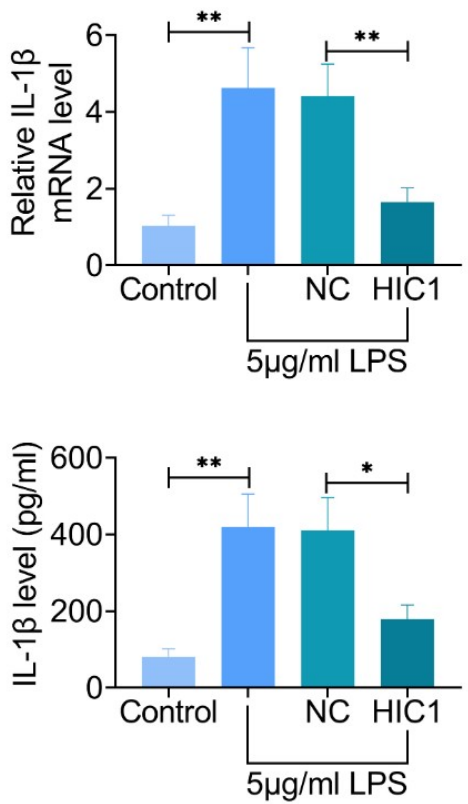

F I G U R E 3. Overexpression of HIC1 attenuated LPS-induced inflammation in HK-2 cell line. (A) QPCR results and (B) ELISA results of TNF- $\alpha$, IL-6, and IL- $1 \beta$ expressions in LPS-treated HK-2 cell line. $* p<0.05$ and $* * p<0.01 v s$. control or LPS + HIC. Data are expressed as mean \pm SEM.
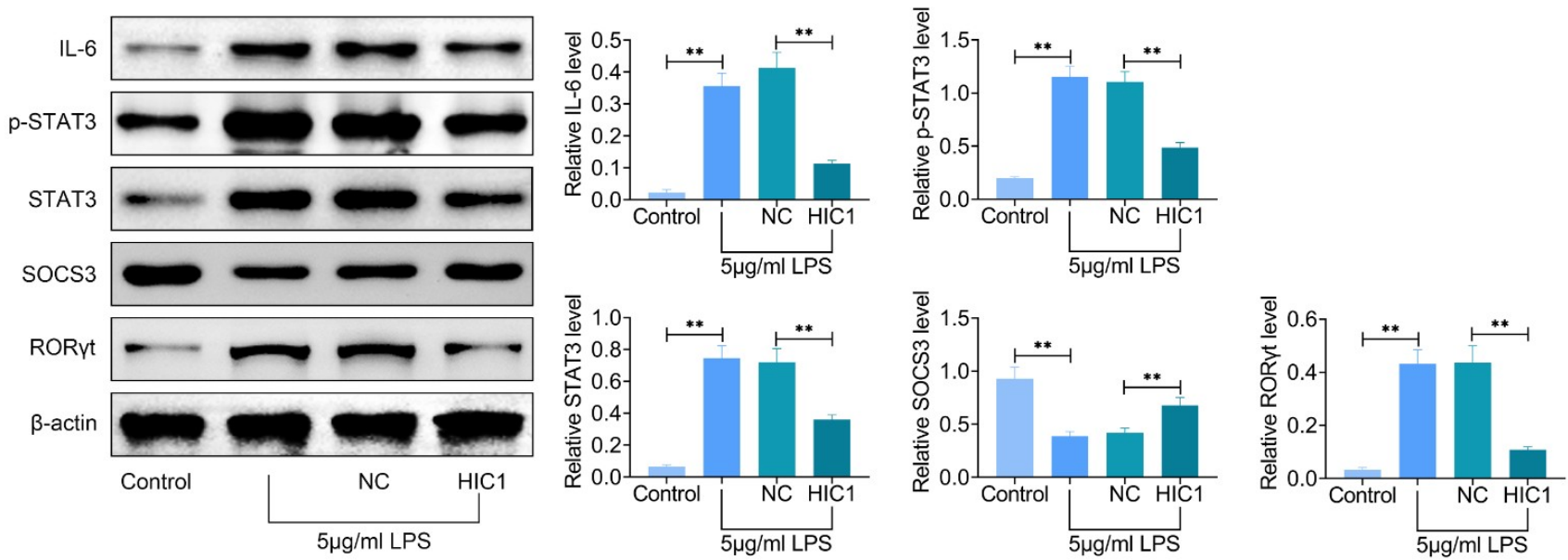

F I G U R E 4. HIC1 regulated LPS-induced injury in HK-2 cell line via regulating IL-6/STAT3 signaling pathway. Western blot analysis and quantitative data for STAT3, p-STAT3, IL-6, ROR $\gamma \mathrm{t}$ and SOCS3 expression in LPS-treated HK-2 cell line. * $p$ $<0.05$ and $* * p<0.01$ vs. control or LPS + HIC. Data are expressed as mean \pm SEM.

In addition, LPS could be recognized by Toll-like receptors and $\mathrm{NF}-\kappa \mathrm{B}$ signaling pathway, a critical pathway of all the signal transduction pathways mediated by LPS, indicating that LPS may be an essential target to enhance the pro-inflammatory response and cell injury [30,31]. Indeed, this study suggested that the protein expression levels of proinflammatory cytokines (TNF- $\alpha$, IL-6, and IL-1 $\beta$ ) were increased by LPS treatment. Conversely, HICl overexpression markedly inhibited the LPSinduced inflammatory response in $\mathrm{HK}-2$ cell line.

Numerous studies have reported that IL-6 and its major effector STAT3 are pro-tumorigenic agents in many cancers and are critical in managing essential cellular progressions, such as cell proliferation, differentiation, inflammation, angiogenesis, and metastasis [32, 33]. The activation of STAT3 was involved in the progression of oncogenic transformation and tumor formation. Recent studies have indicated that netrin1 protected kidney function is dependent on the inhibition of IL6/STAT3 pathway [34]. Moreover, the suppression of renal fibrosis and pentraxin 3 induced STAT3 activation is mediated by IL-6 [35]. Previous studies reported that STAT3 is one of 
the $H I C 1$-interacting proteins and $H I C l$ also suppressed the STAT3-mediated reporter activity [36]. STAT3 is regularly triggered in a wide range of human cancers, and hypermethylation of $\mathrm{HICl}$ promoter in most cancers may increase the STAT3 activity and accordingly promote oncogenesis [27]. In non-small cell lung cancer, HICl was found to be a suppressor of IL-6, which could in turn inhibit HIC1 expression [37, 38]. Promoting HIC1 expression or HIC1-STAT3 interaction may provide a potential therapeutic strategy in antagonizing STAT3-associated human cancers. From this result, it can be concluded that the expressions of HICl and IL-6 could be reciprocally regulated by each other. However, previous studies have focused on its regulatory relationship in cancer research, and there has not been reported the mechanism of IL6 expression inhibited by $\mathrm{HICl}$ in renal cell injury. This is the first study showing that $\mathrm{HICl}$ could inhibit the LPS-induced expressions of STAT3, p-STAT3, IL-6, and ROR $\gamma$ t in HK-2 cell line. A limitation of this study is that only study on the cell-based level, the animal study will be the direction of the future.

\section{Conclusion}

In summary, these results suggested that overexpression of HICl could promote viability and suppress apoptosis and inflammatory response in the LPS-treated HK-2 cell line. Furthermore, the protective effect of $\mathrm{HICl}$ overexpression was partly generated through the inhibition of IL-6/STAT3 signaling pathways. HICl and IL-6/STAT3 pathway are essential to elucidate the mechanism of renal cell injury, and these findings may help to identify the promising targets for clinical sepsis therapy.

\section{AUTHOR CONTRIBUTIONS}

$\mathrm{XY}, \mathrm{JFG}$ - designed the study, supervised the data collection; XSC - analyzed the data, interpreted the data; YJY — prepared the manuscript for publication and reviewed the draft of the manuscript, all authors have read and approved the manuscript.

\section{ETHICS APPROVAL AND CONSENT TO PARTICIPATE}

Not applicable.

\section{ACKNOWLEDGMENT}

Thanks to all the peer reviewers for their opinions and suggestions.

\section{FUNDING}

This research received no external funding.

\section{CONFLICT OF INTEREST}

The authors declare no conflict of interest.

\section{REFERENCES}

[1] Arina P, Singer M. Pathophysiology of sepsis. Current Opinion in Anaesthesiology. 2021; 34: 77-84.

[2] Schlapbach LJ, Trück J, Roger T. The immunology of sepsisunderstanding host susceptibility, pathogenesis of disease, and avenues for future treatment. Frontiers in Immunology. 2020; 11: 1263.

[3] Mayeux PR, MacMillan-Crow LA. Pharmacological targets in the renal peritubular microenvironment: implications for therapy for sepsisinduced acute kidney injury. Pharmacology \& Therapeutics. 2012; 134 : 139-155.

[4] Gonsalez SR, Cortês AL, Silva RCD, Lowe J, Prieto MC, Silva Lara LD. Acute kidney injury overview: from basic findings to new prevention and therapy strategies. Pharmacology \& Therapeutics. 2019; 200: 1-12.

[5] Zarjou A, Agarwal A. Sepsis and acute kidney injury. Journal of the American Society of Nephrology. 2011; 22: 999-1006.

[6] White LE, Hassoun HT. Inflammatory mechanisms of organ crosstalk during ischemic acute kidney injury. International Journal of Nephrology. 2012; 2012: 505197.

[7] Tan RZ, Wang C, Deng C, Zhong X, Yan Y, Luo Y, et al. Quercetin protects against cisplatin-induced acute kidney injury by inhibiting Mincle/Syk/NF- $\kappa \mathrm{B}$ signaling maintained macrophage inflammation. Phytotherapy Research. 2020; 34: 139-152.

[8] Inagi R. Endoplasmic reticulum stress as a progression factor for kidney injury. Current Opinion in Pharmacology. 2010; 10: 156-165.

[9] Gomez H, Ince C, De Backer D, Pickkers P, Payen D, Hotchkiss $\mathrm{J}$, et al. A unified theory of sepsis-induced acute kidney injury inflammation, microcirculatory dysfunction, bioenergetics, and the tubular cell adaptation to injury. Shock. 2014; 41: 3-11.

[10] Fleuriel C, Touka M, Boulay G, Guérardel C, Rood BR, Leprince D. HIC1 (Hypermethylated in Cancer 1) epigenetic silencing in tumors. The International Journal of Biochemistry \& Cell Biology. 2009; 41: 26-33.

[11] Briones VR, Chen S, Riegel AT, Lechleider RJ. Mechanism of fibroblast growth factor-binding protein 1 repression by TGF-beta. Biochemical and Biophysical Research Communications. 2006; 345: 595-601.

[12] Tseng RC, Lee CC, Hsu HS, Tzao C, Wang YC. Distinct HIC1-SIRT1p53 loop deregulation in lung squamous carcinoma and adenocarcinoma patients. Neoplasia. 2009; 11: 763-770.

[13] Kim MK, Jeon BN, Koh DI, Kim KS, Park SY, Yun CO, et al. Regulation of the cyclin-dependent kinase inhibitor $1 A$ Gene $(C D K N 1 A)$ by the repressor BOZF1 through inhibition of $p 53$ acetylation and transcription factor Sp1 binding. Journal of Biological Chemistry. 2013; 288: 70537064.

[14] Hu B, Zhang K, Li S, Li H, Yan Z, Huang L, et al. HIC1 attenuates invasion and metastasis by inhibiting the IL-6/STAT3 signalling pathway in human pancreatic cancer. Cancer Letters. 2016; 376: 387-398.

[15] Zeng S, Wu X, Chen X, Xu H, Zhang T, Xu Y. Hypermethylated in cancer 1 (HIC1) mediates high glucose induced ROS accumulation in renal tubular epithelial cells by epigenetically repressing SIRT1 transcription. Biochimica et Biophysica Acta (BBA)-Gene Regulatory Mechanisms. 2018; 1861: 917-927.

[16] Shuai K. Modulation of STAT signaling by STAT-interacting proteins. Oncogene. 2000; 19: 2638-2644.

[17] Yang J, Stark GR. Roles of unphosphorylated STATs in signaling. Cell Research. 2008; 18: 443-451.

[18] Morris R, Kershaw NJ, Babon JJ. The molecular details of cytokine signaling via the JAK/STAT pathway. Protein Science. 2018; 27: 1984 2009.

[19] Sirkisoon SR, Carpenter RL, Rimkus T, Anderson A, Harrison A, Lange AM, et al. Interaction between STAT3 and GLI1/tGLI1 oncogenic transcription factors promotes the aggressiveness of triple-negative breast cancers and HER2-enriched breast cancer. Oncogene. 2018; 37: 25022514.

[20] Unver N, McAllister F. IL-6 family cytokines: key inflammatory mediators as biomarkers and potential therapeutic targets. Cytokine \& Growth Factor Reviews. 2018; 41: 10-17.

[21] Wang L, Cao L, Wang H, Liu B, Zhang Q, Meng Z, et al. Cancerassociated fibroblasts enhance metastatic potential of lung cancer cells through IL-6/STAT3 signaling pathway. Oncotarget. 2017; 8: 7611676128. 
[22] Ning F, Zheng H, Tian H, Wang T, Hao D, Han S, et al. Research on effect of adiponectin on sepsis-induced lung injury in rats through IL-6/STAT3 signaling pathway. Panminerva Medica. 2020; 62: 184-186.

[23] Li Y, Yao M, Wu T, Zhang L, Wang Y, Chen L, et al. Loss of hypermethylated in cancer 1 (HICl) promotes lung cancer progression. Cellular Signalling. 2019; 53: 162-169.

[24] Pianta TJ, Gobe GC, Owens EP, Endre ZH. Overview of pathophysiology of acute kidney injury: human evidence, mechanisms, pathological correlations and biomarkers and animal models. In: Waikar S., Murray P., Singh A. (eds) Core Concepts in Acute Kidney Injury (pp. 45-67). 1st edn. Springer: New York, NY. 2018.

[25] Chen WY, Wang DH, Yen RC, Luo J, Gu W, Baylin SB. Tumor suppressor HIC1 directly regulates SIRT1 to modulate p53-dependent DNA-damage responses. Cell. 2005; 123: 437-448.

[26] Liao Y, Guo S, Chen Y, Cao D, Xu H, Yang C, et al. VSIG4 expression on macrophages facilitates lung cancer development. Laboratory Investigation. 2014; 94: 706-715.

[27] Lin YM, Wang CM, Jeng JC, Leprince D, Shih HM. HIC1 interacts with and modulates the activity of STAT3. Cell Cycle. 2013; 12: 2266-2276.

[28] Wu W, Zhang L, Lin J, Huang H, Shi B, Lin X, et al. Hypermethylation of the HIC1 promoter and aberrant expression of HIC1/SIRT1 contribute to the development of thyroid papillary carcinoma. Oncotarget. 2016; 7: 84416-84427.

[29] Kuwabara T, Imajoh-Ohmi S. LPS-induced apoptosis is dependent upon mitochondrial dysfunction. Apoptosis. 2004; 9: 467-474.

[30] Liu S, Gallo DJ, Green AM, Williams DL, Gong X, Shapiro RA, et al. Role of toll-like receptors in changes in gene expression and NF-kappa $\mathrm{B}$ activation in mouse hepatocytes stimulated with lipopolysaccharide. Infection and Immunity. 2002; 70: 3433-3442.

[31] Lin M, Rikihisa Y. Ehrlichia chaffeensisdownregulates surface Tolllike receptors 2/4, CD14 and transcription factors PU.1 and inhibits lipopolysaccharide activation of NF-kappa B, ERK 1/2 and p38 MAPK in host monocytes. Cellular Microbiology. 2004; 6: 175-186.
[32] Li N, Grivennikov SI, Karin M. The unholy trinity: inflammation, cytokines, and STAT3 shape the cancer microenvironment. Cancer Cell. 2011; 19: 429-431.

[33] Gupta S, Jain A, Syed SN, Snodgrass RG, Pflüger-Müller B, Leisegang MS, et al. IL-6 augments IL-4-induced polarization of primary human macrophages through synergy of STAT3, STAT6 and BATF transcription factors. Oncoimmunology. 2018; 7: e1494110.

[34] Ranganathan P, Jayakumar C, Ramesh G. Proximal tubule-specific overexpression of netrin-1 suppresses acute kidney injury-induced interstitial fibrosis and glomerulosclerosis through suppression of IL6/STAT3 signaling. American Journal of Physiology-Renal Physiology. 2013; 304: F1054-F1065.

[35] Xiao Y, Yang N, Zhang Q, Wang Y, Yang S, Liu Z. Pentraxin 3 inhibits acute renal injury-induced interstitial fibrosis through suppression of IL6/Stat3 pathway. Inflammation. 2014; 37: 1895-1901.

[36] Laudisi F, Cherubini F, Monteleone G, Stolfi C. STAT3 interactors as potential therapeutic targets for cancer treatment. International Journal of Molecular Sciences. 2018; 19: 1787.

[37] Wang X, Wang Y, Xiao G, Wang J, Zu L, Hao M, et al. Hypermethylated in cancer 1(HIC1) suppresses non-small cell lung cancer progression by targeting interleukin-6/Stat3 pathway. Oncotarget. 2016; 7: 30350 30364.

[38] Sun X, Qu Q, Lao Y, Zhang M, Yin X, Zhu H, et al. Tumor suppressor $\mathrm{HICl}$ is synergistically compromised by cancer-associated fibroblasts and tumor cells through the IL-6/pSTAT3 axis in breast cancer. BMC Cancer. 2019; 19: 1180 .

How to cite this article: Xiang Yang, Jianfeng Gong, Xiaosheng Cai, Youjun Yuan. Overexpression of HIC1 plays a protective effect on renal cell injury caused by lipopolysaccharide by inhibiting IL-6/STAT3 pathway. Signa Vitae. 2022; 18(2): 147153. doi:10.22514/sv.2022.016. 